\title{
Total pancreatectomy: how, when and why?
}

\author{
Massimo Falconi ${ }^{1,2}$
}

Received: 2 June 2021 / Accepted: 14 July 2021 / Published online: 20 July 2021

(C) Italian Society of Surgery (SIC) 2021

In the 1940s and 1950s, morbidity and mortality rates after partial pancreatectomy were so high that the survival following pancreaticoduodenectomy (PD) could be considered as a Russian roulette. In this context, total pancreatectomy (TP) was introduced with a twofold goal. First, by removing the entire pancreatic gland, surgeons of those times thought that postoperative morbidity and mortality could decrease. In fact, the starting point of this belief was usually related to the action of pancreatic juice from the pancreatic remnant. Second, recurrence rate following partial pancreatectomy was very high and TP was considered a more radical procedure to treat pancreatic malignancies. Actually, the results of TP were disappointing. Oncologic outcomes remained poor and even postoperative morbidity and mortality did not significantly improve. Although, the problem of pancreatic fistula was neutralized, other issues emerged including brittle insulin-dependent diabetes, which was difficult to control in many of these patients, and severe malabsorption due to the loss of exocrine pancreatic secretion. Therefore, TP was almost completely abandoned [1].

The outlook of pancreatic surgery has significantly evolved in recent years. Postoperative morbidity and mortality after pancreatectomy have decreased with mortality rates below $5 \%$ in high-volume referral centers, even after TP. The development of modern pancreatic enzyme supplementation and improvements in diabetes control allow a good control of postoperative exocrine and endocrine insufficiency following partial and total pancreatectomies [2]. The diagnosis of pathologic conditions (i.e., intraductal papillary mucinous neoplasms) that may affect the entire pancreatic gland has also made many steps forward. The oncologic results after the multimodal treatment of pancreatic cancer are nowadays

Massimo Falconi

falconi.massimo@hsr.it

1 School of Medicine, Vita Salute San Raffaele University, Milan, Italy

2 Division of Pancreatic Surgery, IRCCS Ospedale San Raffaele, Milan, Italy better than in the past, thus justifying extended resections up to TP for some patients. Finally, TP may be preferred to partial pancreatectomy in patients with a potential high-risk anastomosis or for the treatment of life-threating postoperative complications.

Therefore, TP should be considered an integral part of the technical skills of every pancreatic surgeon [3, 4]. In this light, Updates in Surgery, collected in a monothematic section four papers that represent an up-to-date overview of the most relevant issues related to TP. Unfortunately, most of the data reported and discussed come from retrospective papers-thus limiting the level of evidence of any recommendation. Despite this intrinsic limitation of current surgical literature, the reader can increase his own personal knowledge of the possible indications of a more and more popular surgical procedure.

In the first paper, Casadei et al. describe [5] the contemporary indications of upfront TP. The decision whether to perform an immediate TP instead of partial pancreatectomy with frozen section examination of the pancreatic margin is not easy. The paper, after a general overview of TP, analyzes the indications of upfront TP for intraductal papillary mucinous neoplasms (IPMNs), pancreatic ductal adenocarcinoma (PDAC), neuroendocrine tumors (NET) and metastatic disease to the pancreas. TP in the setting of vascular resections for PDAC are also discussed. The second paper by Crippa et al. [6] discusses the indications to TP when a "positive" neck margin if found at intraoperative histological examination after partial pancreatectomy. The methodology and accuracy of frozen section (FS) analysis is reported by analyzing the relatively limited data available from the literature. Then Authors focus on the role, limitations and advantages of FS analysis and eventual extension of surgical resection up to TP in patients with NET, IPMNs and PDAC. A management algorithm for surgical treatment of patients with IPMNs including intraoperative strategies is provided. The paper ends with a critical evaluation of the role of FS analysis and TP in patients with PDAC, a highly debated issue with conflicting results coming from retrospective studies. Salvia and colleagues [7] critically analyze the role 
of total pancreatectomy (TP) to prevent the development of postoperative pancreatic fistula (POPF) in patients with high-risk pancreas. They also entered the slippery ground of completion total pancreatectomy $(\mathrm{CP})$ in the management of postoperative severe POPF- a difficult issue with very limited data from the literature. Finally, long-term functional outcomes and quality of life following TP are reviewed by Balzano et al. [8]. These authors also analyzed the role of islet cells auto transplantation (IAT) as a mitigation strategy to reduce impact of TP on endocrine function. Historical background and technical aspects of IAT are discussed as well as IAT for conditions other than chronic pancreatitis and TP with IAT for patients at high risk of developing POPF.

Most surgeons have historically been reluctant to perform TP because of not negligible postoperative morbidity and long-term outcomes with a specific focus on insulin-dependent brittle diabetes. Nowadays, pancreatic surgeons should not fear TP. Obviously, the recommendations made for any pancreatic resection apply even more to total pancreatectomy: careful patients' selection, prehabilitation if needed, patients' education to face postoperative exocrine insufficiency and brittle diabetes, centralization of cases in experienced high-volume hospitals. This latter point is mandatory not only to better manage postoperative complications, but also to support in the best possible way patients, who will certainly undergo a big change in their life.

\section{Author contributions MF drafted the article.}

Funding No funding was received to assist with the preparation of this manuscript.

Availability of data and materials Data sharing not applicable to this article as no datasets were generated or analyzed during the current study.

Code availability Not applicable.

\section{Declarations}

Conflict of interest The authors have no conflicts of interest to declare that are relevant to the content of this article.
Research involving human participants and/or animals Studies with human participants performed by the authors were in accordance with the San Raffaele Scientific Institute Review Board.

Informed consent Informed consent was obtained from all patients included in the studies.

\section{References}

1. ReMine WH, Priestley JT, Judd ES, King JN (1970) Total pancreatectomy. Ann Surg 172(4):595-604. https://doi.org/10.1097/ 00000658-197010000-00006

2. Billings BJ, Christein JD, Harmsen WS, Harrington JR, Chari ST, Que FG, Farnell MB, Nagorney DM, Sarr MG (2005) Quality-oflife after total pancreatectomy: is it really that bad on long-term follow-up? J Gastrointest Surg 9(8):1059-1066. https://doi.org/ 10.1016/j.gassur.2005.05.014 (discussion 1066-7)

3. Hartwig W, Gluth A, Hinz U, Bergmann F, Spronk PE, Hackert T, Werner J, Büchler MW (2015) Total pancreatectomy for primary pancreatic neoplasms: renaissance of an unpopular operation. Ann Surg 261(3):537-546. https://doi.org/10.1097/SLA.0000000000 000791

4. Crippa S, Tamburrino D, Partelli S, Salvia R, Germenia S, Bassi C, Pederzoli P, Falconi M (2011) Total pancreatectomy: indications, different timing, and perioperative and long-term outcomes. Surgery 149(1):79-86. https://doi.org/10.1016/j.surg.2010.04.007

5. Casadei R, Ricci C, Ingaldi C, Alberici L, Minni F (2021) Contemporary indications for upfront total pancreatectomy. Updates Surg (in press)

6. Crippa S, Belfiori G, Tamburrino D, Partelli S, Falconi M (2021) Indications to total pancreatectomy for positive neck margin after partial pancreatectomy: a review of a slippery ground. Updates Surg (in press)

7. Salvia R, Lionetto G, Perri G, Malleo G, Marchegiani G, Paiella S, Bassi C (2021) Total pancreatectomy and pancreatic fistula: friend or foe? Updates Surg (in press)

8. Aleotti F, Nano R, Piemonti L, Falconi M, Balzano G. Total pancreatectomy sequelae and quality of life: results of islet autotransplantation as a possible mitigation strategy. Updates Surg (in press)

Publisher's Note Springer Nature remains neutral with regard to jurisdictional claims in published maps and institutional affiliations. 